\title{
The Pharmacogenetics Laboratory of the Department of Pharmacology and Toxicology at the American University of Beirut Faculty of Medicine
}

\begin{abstract}
The pharmacogenetics (PGx) laboratory at the Department of Pharmacology and Toxicology at the American University of Beirut Faculty of Medicine was established in October 2007. Several projects on the genetic polymorphisms of drug metabolizing enzymes and transporters with treatment of noncommunicable diseases such as cardiac diseases and cancers are ongoing. We have been applying the 'candidate gene' PGx approach, and recently started using higher throughput analyses. The more recent research projects are geared towards performing more extensive genotyping and including bigger and more representative population samples such as by developing research registries and prospectively following up patients. Furthermore, many technologies and research applications, such as next-generation sequencing and pharmacoepigenetics that complement and enhance PGx research and applications, are being actively pursued.
\end{abstract}

First draft submitted: 7 July 2017; Accepted for publication: 14 July 2017; Published online: 23 August 2017

Keywords: American University of Beirut Faculty of Medicine • Lebanon

- pharmacoepigenetics $\bullet$ pharmacogenetics

The pharmacogenetics (PGx) laboratory (lab) at the Department of Pharmacology and Toxicology at the American University of Beirut Faculty of Medicine (AUBFM) was established in October 2007 upon the recruitment of Dr Nathalie K Zgheib, a Family Physician, graduate of the American University of Beirut (AUB) who trained in PGx, drug metabolism and interindividual variability at the Center for Clinical Pharmacology at the University of Pittsburgh with Drs Robert A Branch and Marjorie Romkes.

Since then, the PGx lab has been gradually and steadily growing, and has attracted increasing numbers of collaborators from the clinical departments, such that currently, there are at least 6000 biologic samples stored and available for research (Figure 1 \& Supplementary Table 1). The PGx lab is pursuing two main lines of research: applied pharmacogenetic and pharmacoepigenetic research and translational and clinical utility of $\mathrm{PGx}$, while also being invested in teaching PGx.

\section{Applied PGx \& pharmacoepigenetic research \\ Cardiology}

Given that people in the Middle East have different genetic makeup and associated phenotypes when compared with western populations, we first investigated the effect of CYP2C9 and VKORC1 genetic polymorphisms on oral anticoagulants as a proof of concept project [1]. Predicting the dose of anticoagulants was more accurate when we used Artificial Neural Networks (ANN) instead of regression analysis [2], so we developed an online application that integrates the ANN model to calculate the oral anticoagulant dose needed for a specific
Nathalie K Zgheib

Department of Pharmacology \& Toxicology, American University of Beirut Faculty of Medicine, Beirut, Lebanon Tel.: +961 1350 000; ext: 4846 nk16@aub.edu.lb 
Institutional Profile Zgheib

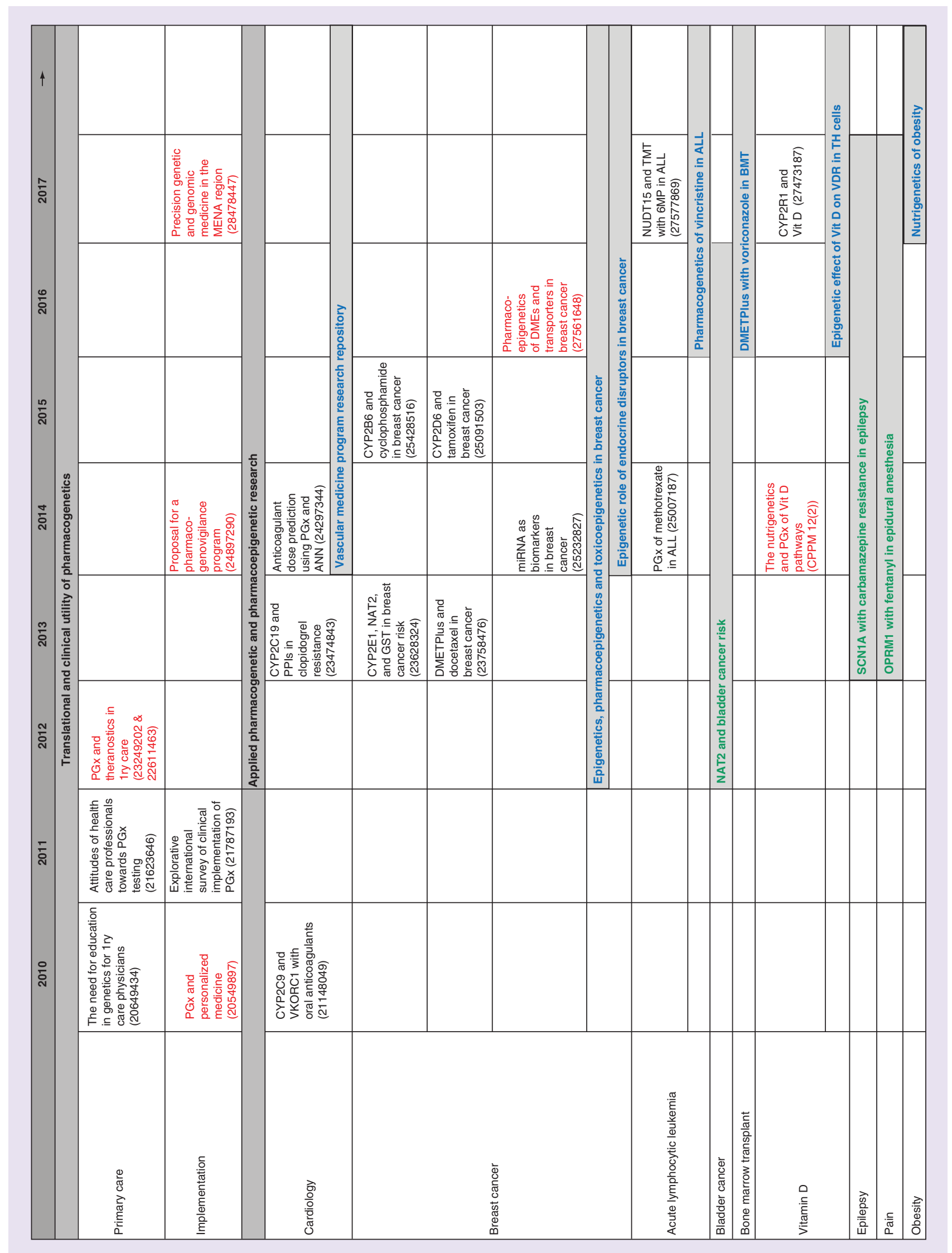


Figure 1. Timeline of select PGx research at the PGx lab of the Department of Pharmacology and Toxicology, American University of Beirut Faculty of Medicine (see previous page). Black font refers to published original research articles. Red font refers to published review or perspective articles. Green font refers to completed projects with manuscripts currently being written or under review. Blue font refers to ongoing projects. Numbers between parentheses refer to the PubMed ID.

ALL: Acute lymphocytic leukemia; BMT: Bone marrow transplant; DMEs: Drug metabolizing enzymes; PGx: Pharmacogenetics; Vit D: Vitamin D.

For color figures, please see online at https://www.futuremedicine.com/doi/10.2217/pgs-2017-0122

target International Normalized Ratio (INR). The lab also evaluated the PGx of clopidogrel and concluded that CYP2C19 genetic polymorphisms do not play a major role in clopidogrel resistance in the Lebanese [3]. The lab is currently leading the biologic repository of the vascular medicine program at AUBFM where patients admitted to the Cardiac Catheterization Lab and Coronary Care unit at AUB Medical Center are recruited. The repository includes blood and urine collection that is linked to medical information including medication use, lab values and cardiac end points. The repository will be an excellent resource for translational research questions.

\section{Cancer}

The PGx lab has been involved in cancer genomics and established a repository (blood and tissue samples and clinical data) for breast cancer patients. The candidate SNPs approach in drug metabolizing enzymes (DMEs) was first adopted to evaluate potential associations with breast cancer risk [4] and interindividual variability of cyclophosphamide based cytotoxic therapy [5]. Using high-throughput analyses, the PGx lab was the first in the Middle East to use the DMEs and transporters array on the Affymetrix platform in order to assess for the effect of genetic polymorphisms in DMEs and transporters on docetaxel toxicity [6]. The role of an array of CYP2D6 genetic polymorphisms, among other DMEs and in partnership with international collaborators, was then evaluated in relation to tamoxifen hormonal therapy [7].

We took advantage of the breast cancer repository dataset to investigate epigenetic factors that may play a role in individualized therapy (pharmacoepigenetics). Pilot data on levels of tissue and circulating miRNA in Lebanese breast cancer patients have been generated [8], and it is hoped that specific miRNAs may be used as biomarkers for the efficacy or toxicity of therapy in breast cancer [9]. Additional toxicoepigenetic research is ongoing on the role of $A B C B 1$ promoter methylation in peripheral blood with docetaxel related neutropenia. The lab is also currently part of a multidisciplinary toxicology project investigating the role and mechanism of environmental toxins, mainly Bisphenols A, F and S in breast cancer. This project uses the breast cancer repository in addition to a newly established, very well phenotyped cohort of Lebanese people living in Greater Beirut area. All participants have undergone physical exam for anthropometric measurements, extensive blood testing for metabolic and other abnormalities, and a food frequency questionnaire. Accordingly, candidate SNPs analysis for a nutrigenetics project is also in progress.

The PGx lab also used its expertise to determine the effect of candidate SNPs on toxicity from methotrexate [10], 6-mercaptopurine [11] and vincristine therapy in children with acute lymphocytic leukemia. Other ongoing PGx projects in cancer include the distribution and role of NAT2 genetic polymorphisms in bladder cancer risk, and the role of genetic polymorphisms of DMEs and transporters in the interaction between voriconazole and cyclosporine in patients undergoing bone marrow transplantation.

\section{Other}

Other collaborative PGx research projects are ongoing to include the role of candidate SNPs in OPRM1 in response to epidural fentanyl analgesia. The PGx lab is also part of a collaborative effort in storing DNA and blood for a large number of very well phenotyped patients with newly diagnosed epilepsy and who are being prospectively followed up for treatment outcome. An ongoing project on the potential role of a SCN1A candidate SNP in resistance to carbamazepine builds on these data collection. The PGx lab was also involved in the evaluation of CYP2R1 genetic polymorphisms as potential drivers for the reportedly low levels of 25-hydroxy vitamin D in the Lebanese despite the plentiful sunshine, and whether these polymorphisms affect response to oral vitamin D supplementation [12]. Further work is in progress on the potential epigenetic effects of vitamin $\mathrm{D}$ on the vitamin $\mathrm{D}$ receptor.

\section{Translational \& clinical utility of PGx}

Despite the important role that physicians are expected to play in PGx-based prescribing to reduce the rates of adverse drug events, the adoption of PGx testing unfortunately remains limited. The PGx lab therefore, and in anticipation of the broader clinical applications of PGx, conducted empiric research on the ethical, social, economic and cultural factors that may influence implementation of PGX-based prescribing in low- and middle-income countries, with a focus on primary care. 
Three original research articles on the underutilization of genetic services by Middle Eastern primary care practitioners [13], the attitudes of healthcare professionals toward PGx testing [14] and a comparison between underdeveloped and developed countries regarding the clinical application of PGx [15] were published. Results of these surveys revealed that physicians' education and buy-in are key to implement the clinical applications of PGx. As a matter of fact at AUB Medical Center, the molecular diagnostics laboratory launched genotyping services for $C Y P 2 C 9$ and $V K O R C 1$ with oral anticoagulants, yet there was very little clinical use of the test, and it was hence discontinued. The same applies to CYP2C19 genotyping especially that, at the time, we genotyped for CYP2C19*2 and *3 only.

We believe that there is still a long way before the implementation of personalized medicine becomes a reality in our region. We shared our insight on the matter by writing review articles and perspectives on the advances of PGx applications, the barriers for their adoption in the clinic and the efforts that are being undertaken to move PGx forward $[16,17]$. Additionally, and in the era of precision medicine, the PGx lab proposes and hopes to foster the establishment of a pharmacogenovigilance program in primary care [18].

\section{Teaching PGx}

The PGx lab (Dr Zgheib) has been involved in teaching PGx to medical students, graduate nursing students as well as graduates and $\mathrm{PhD}$ students of the biomedical sciences at AUBFM. An innovative and efficient teaching method, called Team Based Learning, that is well suited for resource-limited countries and greatly liked by students is employed [19]. Students are challenged with therapeutic scenarios and given an exercise whereby they are asked to find the evidence for a drug's dosing based on PGx data using the Pharmacogenomics Knowledge Base website, and to review and analyze the recommendations of the clinical PGx implementation consortium. The PGx lab has also conducted empiric research on the teaching of PGx in low and middle income countries [20].

\section{Lab support}

The PGx lab has been funded by intramural and extramural grants most of which are collaborative with a number of AUBFM investigators such as cardiologists, hemato-oncologists, urologists, endocrinologists, neurologists, gastroenterologists, pathologists and others. International collaborations to include Europe (such as France and Germany) and the USA have been established. The lab also makes use of instruments available at the AUBFM core lab facilities and the AUBFM infrastructure for research support such as the services of the Clinical Research Institute (CRI) for data management and analysis.

The maintenance of the lab at its highest functional level has also been dependent on its personnel and students who gradually acquire expertise in several PGx and laboratory techniques. This includes conducting patients' consent and recruitment; clinical data collection and entry; samples collection, handling and preparation; optimization, running, analysis and troubleshooting of low- and high-throughput platforms and analyses to include genotyping, RNA and miRNA expression, and methylation; following ethical guidelines and good clinical practice for human subjects research and good laboratory practices; and generating reliable and reproducible data.

\section{Challenges}

Despite the support that the PGx lab has been receiving, its operations and growth have been hindered by several challenges. First and foremost is the limitation in funding; for instance, although there are a number of intramural funding opportunities, many of which have been recently launched by AUB and AUBFM, the maximum allowable amount per year is barely enough to support initiation of project ideas and generation of proof of principle and preliminary data for further extramural funding. On the other hand, securing international large extramural grants for research has been quite difficult. In addition, the cost of lab items in Lebanon is double or triple that in Western countries due to the presence of exclusive dealers for the various companies. Furthermore, the available sample sizes remain relatively low. It is hence believed that the only way to move forward for more translational value and impact is to nurture local, regional and international collaborations. These would hopefully facilitate the collection and pooling of larger sample populations in addition to the sharing of funds, data and scientific knowhow to include new technologies such as next-generation sequencing.

\section{Conclusion \& future perspective}

The PGx lab of the Department of Pharmacology and Toxicology at the AUBFM began by applying the 'candidate gene' PGx approach, and then moved to using higher throughput analyses, but with limited sample sizes. Planned research projects are therefore geared towards performing more extensive genotyping and including bigger and more representative population samples such as by developing research registries and prospectively following up patients. 
Furthermore, many technologies and research applications, such as next-generation sequencing and pharmacoepigenetics that complement and enhance PGx research and applications, are being actively pursued. It is hoped that with more time, perseverance, additional funds and collaborations, the PGx lab will continue to further develop PGx research in Lebanon and the region.

\section{Acknowledgements}

AUBFM core labs, collaborating faculty and labs, previous and current students, research assistants and research fellows of the PGx lab, research participants. The author would also like to acknowledge Drs R Sabra and S Khoury for revising the manuscript.

\section{Financial \& competing interests disclosure}

The author received funding from the University Research Board (URB), American University of Beirut Faculty of Medicine
(AUBFM) Medical Practice Plan (MPP) grants and the Lebanese National Council for Scientific Research (LNCSR). The author has no other relevant affiliations or financial involvement with any organization or entity with a financial interest in or financial conflict with the subject matter or materials discussed in the manuscript apart from those disclosed.

No writing assistance was utilized in the production of this manuscript.

\section{Supplementary data}

To view the supplementary data that accompany this paper please visit the journal website at: www.futuremedicine.com/ doi/full/10.2217/pgs-2017-0122

\section{Open access}

This work is licensed under the Attribution-NonCommercialNoDerivatives 4.0 Unported License. To view a copy of this license, visit http://creativecommons.org/licenses/by-nc$\mathrm{nd} / 4.0 /$

\section{References}

1 Esmerian MO, Mitri Z, Habbal MZ et al. Influence of CYP2C9 and VKORC1 polymorphisms on warfarin and acenocoumarol in a sample of Lebanese people. J. Clin. Pharmacol. 51(10), 1418-1428 (2011).

2 Isma'eel HA, Sakr GE, Habib RH, Almedawar MM, Zgheib NK, Elhajj IH. Improved accuracy of anticoagulant dose prediction using a pharmacogenetic and artificial neural network-based method. Eur. J. Clin. Pharmacol. 70(3), 265-273 (2014).

3 El-Halabi MM, Zgheib N, Mansour NM et al. CYP2C19 genetic polymorphism, rabeprazole and esomeprazole have no effect on the antiplatelet action of clopidogrel. J. Cardiovasc. Pharmacol. 62(1), 41-49 (2013).

4 Zgheib NK, Shamseddine AA, Geryess E et al. Genetic polymorphisms of CYP2E1, GST, and NAT2 enzymes are not associated with risk of breast cancer in a sample of Lebanese women. Mutat. Res. 747-748, 40-47 (2013).

5 Haroun F, Al-Shaar L, Habib RH et al. Effects of CYP2BG genetic polymorphisms in patients receiving cyclophosphamide combination chemotherapy for breast cancer. Cancer Chemother. Pharmacol. 75(1), 207-214 (2015).

6 Awada Z, Haider S, Tfayli A et al. Pharmacogenomics variation in drug metabolizing enzymes and transporters in relation to docetaxel toxicity in Lebanese breast cancer patients: paving the way for OMICs in low and middle income countries. OMICS 17(7), 353-367 (2013).

7 Saladores P, Murdter T, Eccles D et al. Tamoxifen metabolism predicts drug concentrations and outcome in premenopausal patients with early breast cancer. Pharmacogenomics J. 15(1), 84-94 (2015).

8 Nassar FJ, El SM, Zgheib NK et al. miRNA as potential biomarkers of breast cancer in the Lebanese population and in young women: a pilot study. PLoS ONE 9(9), e107566 (2014).
9 Nasr R, Sleiman F, Awada Z, Zgheib NK. The pharmacoepigenetics of drug metabolism and transport in breast cancer: review of the literature and in silico analysis. Pharmacogenomics 17(14), 1573-1585 (2016).

10 Zgheib NK, Akra-Ismail M, Aridi C et al. Genetic polymorphisms in candidate genes predict increased toxicity with methotrexate therapy in Lebanese children with acute lymphoblastic leukemia. Pharmacogenet. Genomics 24(8), 387-396 (2014).

11 Zgheib NK, Akika R, Mahfouz R et al. NUDT15 and TPMT genetic polymorphisms are related to 6-mercaptopurine intolerance in children treated for acute lymphoblastic leukemia at the Children's Cancer Center of Lebanon. Pediatr. Blood Cancer 64(1), 146-150 (2017).

12 Arabi A, Khoueiry-Zgheib N, Awada Z et al. CYP2R1 polymorphisms are important modulators of circulating 25-hydroxyvitamin D levels in elderly females with vitamin insufficiency, but not of the response to vitamin D supplementation. Osteoporos. Int. 28(1), 279-290 (2017).

13 Antoun J, Zgheib NK, Ashkar K. Education may improve the underutilization of genetic services by Middle Eastern primary care practitioners. Genet. Test Mol. Biomarkers 14(4), 447-454 (2010).

14 Zgheib NK, Arawi T, Mahfouz RA, Sabra R. Attitudes of health care professionals toward pharmacogenetic testing. Mol. Diagn. Ther. 15(2), 115-122 (2011).

15 Ghaddar F, Cascorbi I, Zgheib NK. Clinical implementation of pharmacogenetics: a nonrepresentative explorative survey to participants of WorldPharma 2010. Pharmacogenomics 12(7), 1051-1059 (2011).

16 Bartlett G, Antoun J, Zgheib NK. Theranostics in primary care: pharmacogenomics tests and beyond. Expert. Rev. Mol. Diagn. 12(8), 841-855 (2012). 
17 Bilani N, Dagher M, Zgheib NK. Precision genetic and genomic medicine in the Middle East and North Africa region: are we there yet? Public Health Genomics doi:10.1159/000473890 (2017) (Epub ahead of print).

18 Awada Z, Zgheib NK. Pharmacogenovigilance: a pharmacogenomics pharmacovigilance program. Pharmacogenomics 15(6), 845-856 (2014).
19 Zgheib NK, Simaan JA, Sabra R. Using team-based learning to teach pharmacology to second year medical students improves student performance. Med. Teach. 32(2), 130-135 (2010).

20 Ghaddar F, Saba K, Zgheib NK. Teaching pharmacogenetics in low and middle income countries (LMICS): an empirical study of the lessons learned. Curr. Pharmacogenomics Person. Med. 10(3), 217-225 (2012). 